\title{
VECTORS BUNDLES WITH THETA DIVISORS I BUNDLES ON CASTELNUOVO CURVES
}

\author{
KIRTI JOSHI AND V. B. MEHTA
}

\begin{abstract}
In this paper we show that semistable vector bundles on a Castelnuovo curve of genus $g \geq 2$ have theta divisors. As a corollary, we deduce that semistable vector bundles on a smooth, general curve of genus $g \geq 2$ which extend to semistable vector bundles on any Castelnuovo degeneration of the general curve admit a theta divisor.
\end{abstract}

\section{INTRODUCTION}

1.1. The classical case. Let $C$ be a smooth, projective curve of genus $g \geq 2$. We say, following [Ray82, that a vector bundle $V$ on $C$ with $\mu(V)=\operatorname{deg}(V) / \operatorname{rk}(V)=0$, has a theta divisor if $\operatorname{Hom}\left(L_{\text {gen }}, V\right)=0$ for a general line bundle $L_{\text {gen }}$ of degree $1-g$. When such a line bundle exists, the set of line bundles for which $\operatorname{Hom}(L, V) \neq 0$ forms a divisor in $\operatorname{Pic}^{1-g}(C)$, which is algebraically equivalent to $\mathrm{rk}(V) \theta$ where $\theta$ is the classical theta divisor of $\mathrm{Pic}^{1-g}(C)$. It is easy to see that if $V$ has a theta divisor then $V$ is semistable. It was shown in Ray82 that there are stable vector bundles on a smooth, projective curve of genus $g \geq 2$ which do not have a theta divisor. Raynaud also showed that if $\operatorname{rk}(V)=2$ then every semistable vector bundle has a theta divisor and if $\mathrm{rk}(V)=3$ then the assertion continues to hold if $X$ is generic. It is well-known that locus of semi-stable vector bundles with theta divisors is open in the moduli of semi-stable vector bundles (see Ray82). But at the moment we do not have any concrete description of this locus. In the present note we provide an open set of the moduli of semi-stable vector bundles (on a generic curve) which have theta divisors. In this note we prove the following theorems.

Theorem 1.1.1. Suppose $\mathfrak{X} \rightarrow \operatorname{Spec}(k[[t]])$ is a flat, proper family of curves with smooth generic fibre $\mathfrak{X}_{\eta}$ and the special fibre $\mathfrak{X}_{0}=X$ is a Castelnuovo curve of arithmetic genus $g \geq 2$. Suppose $V$ is a semistable vector bundle on $X_{\eta}$ of degree zero which extends as a semistable vector bundle on $\mathfrak{X}_{0}$. Then $V$ has a theta divisor.

Recall that a projective, irreducible nodal curve $X$ of arithmetic genus $g$ and with $g$ nodes is called a Castelnuovo curve of genus $g$. By the results of [DM69] we know that a smooth, general curve of genus $g$ admits a Castelnuovo degeneration. Thus the above theorem has the following corollary.

Corollary 1.1.2. Let $X / k$ be a general smooth, projective curve of genus $g \geq 2$. Let $V$ be a semistable vector bundle on $X$ which extends to a semistable vector bundle on some Castelnuovo degeneration of $X$. Then $V$ admits a theta divisor.

1.2. Theta divisors for semistable bundles on Castelnuovo curves. Theorem 1.1 .1 is proved by studying vector bundles on a Castelnuovo curve $X$. By the

Date: Version: Aug 2, 2007. 
semi-continuity theorem it is clear that to prove the Theorem 1.1.1 it is sufficient to prove the following theorem.

Theorem 1.2.1. Let $X$ be a Castelnuovo curve of genus $g \geq 2$. Then every semistable vector bundle on $X$ with $\operatorname{deg}(V)=0$ has a theta divisor.

1.3. Theta divisors for generalized parabolic bundles. Vector bundles and more generally torsion-free sheaves on a Castelnuovo curve $X$ can be described as bundles on the normalization

$$
\pi: \tilde{X}=\mathbb{P}^{1} \rightarrow X
$$

with additional structures. There are several such descriptions available (see Ray82, Ses82, Bho92, Bho96]). The description we will use is the one developed in [Bho92, Bho96]. In [Bho92 torsion-free sheaves on a Castelnuovo curve are described in terms of generalized parabolic bundles. The advantage of this description is that the correspondence between bundles on the normalization $\tilde{X}$ (with additional structures) and bundles on $X$ preserves degrees of the underlying bundles. The notion of stability, semistability of bundles extends easily to generalized parabolic bundles. Vector bundles on a Castelnuovo curve $X$ arise from special type of generalized parabolic bundles which we will call generalized parabolic bundles of type $B$. Any generalized parabolic bundle of type $B$ gives rise to a unique (up to isomorphism) vector bundle on $X$ and conversely; moreover semi-stability (resp. stability) is preserved under this correspondence. Theorem 1.2.1 follows from the following

Theorem 1.3.1. Let $a_{i} \neq b_{i}$ be $g \geq 2$ pairs of points on $\mathbb{P}^{1}$. Let $\left(\tilde{V}, F_{i}(\tilde{V})\right)$ be a generalized parabloic bundle of type $B$ with generalized parabolic structures at $\left\{a_{i}, b_{i}\right\}_{1 \leq i \leq g}$. Assume $\left(\tilde{V}, F_{i}(\tilde{V})\right)$ is semistable with $\mu(V)=0$. Then there exists a line bundle $\tilde{L}$ of degree $1-g$ with a generalized parabolic structure $\left(\tilde{L}, F_{i}(\tilde{L})\right)$ of type $B$ such that

$$
\operatorname{Hom}\left(\left(\tilde{L}, F_{i}(\tilde{L})\right),\left(\tilde{V}, F_{i}(\tilde{V})\right)\right)=0 .
$$

In other words, $\left(\tilde{V}, F_{i}(\tilde{V})\right)$ has a theta-divisor.

1.4. We note that in Ray82 it was shown that there exists stable torsion-free sheaves on a Castelnuovo curve of genus $g \geq 2$ which do not have a theta divisor. The moduli space of semistable torsion-free sheaves on a Castelnuovo curve exists (see [Ses82]) and properly contains the locus of locally-free semistable sheaves as an open subset.

1.5. In this paper we have treated the case of generalized parabolic bundles on $\tilde{X}=\mathbb{P}^{1}$, in a forthcoming paper [JM] we will treat the case when $\tilde{X}$ is an aribitrary smooth, projective curve (not neccessarily of genus 0 ). The details are a little more complicated and it was felt by us that, for readability, it would be best to separate them.

1.6. Acknowledgements. This paper was written while the first author was visiting the Tata Institute and the author thanks the institute for hospitality. We are also grateful to Usha Bhosle for conversations and correspondence about generalized parabolic bundles. 


\section{Preliminaries}

2.1. Castelnuouvo curves. Let $k$ be an algebraically closed field. Let $\tilde{X}=\mathbb{P}^{1}$ be the projective line over $k$. Let $a_{i} \neq b_{i}$ be $1 \leq i \leq g$ pairs of $g$ pairs of points $\tilde{X}$. Identifying $a_{i}$ with $b_{i}$ gives us a nodal curve $X$ which is an integral nodal curve of arithmetic genus $g$ and has exactly $g$ nodes. The normalization of $X$ is $\tilde{X}=\mathbb{P}^{1}$ and the normalization map is $\pi: \tilde{X}=\mathbb{P}^{1} \rightarrow X$ which identifies the points $a_{i}$ with $b_{i}$ for $1 \leq i \leq g$.

Conversely, if $X$ is an integral nodal curve of arithmetic genus $g$ and with $g$ nodes, then it arises in the above manner for some $g$ pairs of points $a_{i} \neq b_{i}$.

We will call, an integral, nodal curve with $g$ nodes, and of arithmetic genus $g$, a Castelnuovo curve of genus $g$.

2.2. In what follows, we will need to work with sheaves on $\tilde{X}$ as well as on $X$. So we will adopt the following convention: sheaves on $\tilde{X}$ will be denoted with a on top, while sheaves on $X$ will be denoted without the ${ }^{\sim}$ on top. Thus $\tilde{V}, \tilde{L}$ etc will be sheaves on $\tilde{X}$, and $V, L$ will be sheaves on $X$.

2.3. Stalks and fibers. Let $\tilde{V}, \tilde{W}$ be vector bundles on $\tilde{X}$, let $\tilde{f}: \tilde{W} \rightarrow \tilde{V}$ be morphism of $\mathcal{O}_{\tilde{X}}$-modules. Let $x \in \tilde{X}$ be a point. We will write $V_{\tilde{X}, x}$ for the stalk of $\tilde{V}$ at $x$ and write $\tilde{V}_{x}=V \otimes_{\mathcal{O}_{\tilde{X}, x}} k$ for the fibre at $x$. Recall that a morphism $\tilde{f}: \tilde{W} \rightarrow \tilde{V}$ induces a morphism on the stalks at $x$ and also a morphism $W_{x} \rightarrow V_{x}$ on the fibres (this morphism may be identically zero!).

2.4. Generalized parabolic bundles. We shall use the formalism of generalized parabolic bundles of [Bho92]. Since this is not so well-known we recall facts proved in Bho92, Bho96. A generalized parabolic bundle (a GPB for short) on $\tilde{X}$ is a collection $\left.\left(\tilde{V},\left\{F_{i}(\tilde{V})\right\}_{1 \leq i \leq g}\right\}\right)$ where $\tilde{V}$ is a vector bundle on $\tilde{X}$ and $F_{i}(\tilde{V}) \subset \tilde{V}_{a_{i}} \oplus \tilde{V}_{b_{i}}$ is a subspace. We will simply write $\left(\tilde{V}, F_{i}(\tilde{V})\right)$ instead of $\left.\left(\tilde{V},\left\{F_{i}(\tilde{V})\right\}_{1 \leq i \leq g}\right\}\right)$ for simplicity.

2.5. Morphisms of generalized parabolic bundles. A morphism of generalized parabolic bundles $f:\left(\tilde{W}, F_{i}(\tilde{W})\right) \rightarrow\left(\tilde{V}, F_{i}(\tilde{V})\right)$ is a morphism $f: W \rightarrow V$ of $\mathcal{O}_{\tilde{X}^{-}}$ modules and a map of vector spaces $F_{i}(\tilde{W}) \rightarrow F_{i}(\tilde{V})$ for $1 \leq i \leq g$ such that the diagram

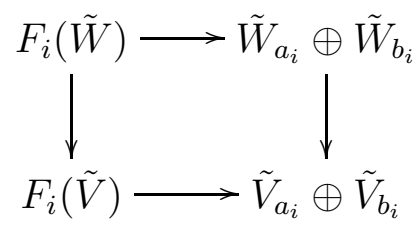

commutes. Here the vertical arrow on the right is the direct sum of the natural arrow (see 2.3) $\tilde{W}_{a_{i}} \rightarrow \tilde{V}_{a_{i}}$ and the corresponding arrow for $b_{i}$.

2.6. Induced GPB structures. Suppose $\left(\tilde{V}, F_{i}(\tilde{V})\right)$ is a GPB and $\tilde{W} \subset \tilde{V}$ is a subsheaf. Then $W$ carries an induced $G P B$ structure: define, for $1 \leq i \leq g$, a 
subspace $F_{i}^{i n d}(\tilde{W}) \subset \tilde{W}_{a_{i}} \oplus \tilde{W}_{b_{i}}$ as the equalizer of the two arrows to $\tilde{V}_{a_{i}} \oplus \tilde{V}_{b_{i}}$ :

$$
\begin{aligned}
& \tilde{W}_{a_{i}} \oplus \tilde{W}_{b_{i}} \\
& F_{i}(\tilde{V}) \longrightarrow \tilde{V}_{a_{i}} \oplus \tilde{V}_{b_{i}}
\end{aligned}
$$

Explicitly $F_{i}^{i n d}(\tilde{W})$ consist of elements of $\tilde{W}_{a_{i}} \oplus \tilde{W}_{b_{i}}$ whose image lies in $F_{i}(\tilde{V})$. The diagram

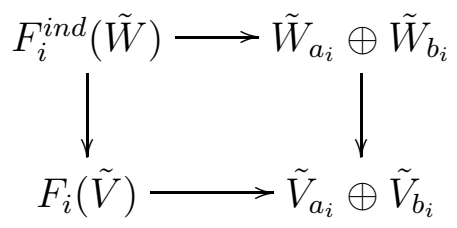

commutes by properties of equalizers. This equips the bundle $\tilde{W}$ with GPB structure, which we call the induced GPB structure on $\tilde{W}$. Obviously we have a morphism $\left(\tilde{W}, F_{i}(\tilde{W})\right) \rightarrow\left(\tilde{V}, F_{i}(\tilde{V})\right)$.

We caution the reader that our definition of GPB structure is slightly different with that of Bho92, where $\tilde{W} \subset \tilde{V}$ is a subbundle, in which case the maps on the fibres is an inclusion, so we can talk about intersections of subspaces of fibres of $\tilde{V}$. It is easy to see that our definition agrees with loc. cit. in this case, and works in all cases.

2.7. Generalized parabolic bundles of types $B$ and $T$. A generalized parabolic bundle $\left(\tilde{V}, F_{i}(\tilde{V})\right)$ is said to be of type $B$ if the composite maps

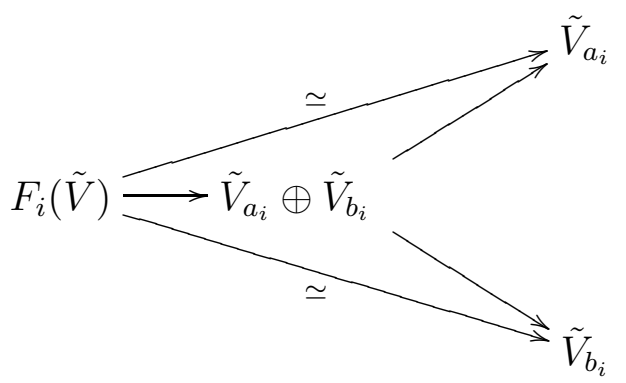

are both isomorphisms. A GPB which is not of type $B$ is called a GPB of type $T$. The terminology has its origins in the nature of the torsion-free sheaves which these bundles give rise to. A GPB of type $B$ gives rise to a locally-free sheaf on $X$, while a GPB of type $T$ gives rise to a torsion-free but non-locally-free sheaf on $X$. Thus type $B$ is short for "bundle type", while type $T$ is everything torsion-free but not bundle type. We caution the reader that this terminology was not introduced in Bho92.

2.8. The following Lemma is immediate from Bho92 (also see Ray82, Ses82).

Lemma 2.8.1. Let $\left(\tilde{V}, F_{i}(\tilde{V})\right)$ be a generalized parabolic bundle on $\tilde{X}$. Then the following are equivalent:

(1) the $G P B\left(\tilde{V}, F_{i}(\tilde{V})\right)$ is of type $B$,

(2) for every $i, 1 \leq i \leq g$, the subspace $F_{i}(\tilde{V}) \subset \tilde{V}_{a_{i}} \oplus \tilde{V}_{b_{i}}$ is the graph of an isomorphism $\tilde{V}_{a_{i}} \rightarrow \tilde{V}_{b_{i}}$. 
2.9. Degrees and slopes. Let $\tilde{V}$ be a vector bundle on $\tilde{X}$. Then its usual degree on $\tilde{X}$ will be $\operatorname{denoted}$ by $\operatorname{deg}(\tilde{V})$, while its slope $\mu(\tilde{V})=\operatorname{deg}(\tilde{V}) / \operatorname{rk}(\tilde{V})$. by

Now let $\left(\tilde{V}, F_{i}(\tilde{V})\right)$ be a GPB on $\tilde{X}$. Then its generalized parabolic degree is given

$$
\operatorname{deg}\left(\left(\tilde{V}, F_{i}(\tilde{V})\right)\right)=\operatorname{deg}(\tilde{V})+\sum_{i=1}^{g} \operatorname{dim} F_{i}(\tilde{V}) .
$$

The generalzed parabolic slope of $\left(\tilde{V}, F_{i}(\tilde{V})\right)$ is a defined by

$$
\mu\left(\left(\tilde{V}, F_{i}(\tilde{V})\right)\right)=\frac{\operatorname{deg}\left(\left(\tilde{V}, F_{i}(\tilde{V})\right)\right)}{\operatorname{rk}(\tilde{V})}=\frac{\operatorname{deg}(\tilde{V})+\sum_{i=1}^{g} \operatorname{dim} F_{i}(\tilde{V})}{\operatorname{rk}(\tilde{V})} .
$$

2.10. Note, in particular, that if $\left(\tilde{V}, F_{i}(\tilde{V})\right)$ is a generalized parabolic bundle of type $B$ and rank $r$ then we have the following formula:

$$
\operatorname{deg}\left(\left(\tilde{V}, F_{i}(\tilde{V})\right)\right)=\operatorname{deg}(\tilde{V})+g r .
$$

Hence we have

$$
\mu\left(\left(\tilde{V}, F_{i}(\tilde{V})\right)\right)=\mu(\tilde{V})+g .
$$

In particular, if $\left(\tilde{L}, F_{i}(\tilde{L})\right)$ is a $\operatorname{GPB}$ of type $B$ with $\operatorname{rk}(\tilde{L})=1, \operatorname{deg}(\tilde{L})=1-g$, then we have

$$
\mu\left(\left(\tilde{L}, F_{i}(\tilde{L})\right)\right)=\operatorname{deg}\left(\left(\tilde{L}, F_{i}(\tilde{L})\right)\right)=1 .
$$

2.11. Stability and semi-stability. We say that a generalized Parabolic bundle $\left(\tilde{V}, F_{i}(\tilde{V})\right)$ is semistable (resp. stable), if for every $\left(\tilde{W}, F_{i}(\tilde{W})\right) \subset\left(\tilde{V}, F_{i}(\tilde{V})\right)$, we have $\mu\left(\left(\tilde{W}, F_{i}(\tilde{W})\right)\right) \leq \mu\left(\left(\tilde{V}, F_{i}(\tilde{V})\right)\right)\left(\operatorname{resp} . \mu\left(\left(\tilde{W}, F_{i}(\tilde{W})\right)\right)<\mu\left(\left(\tilde{V}, F_{i}(\tilde{V})\right)\right)\right)$.

2.12. Torsion-free sheaves on Castelnuovo curves. Let $\left(\tilde{V}, F_{i}(\tilde{V})\right)$ be a GPB on $\tilde{X}$. Then the subsheaf $V$ defined by the exact sequence

$$
0 \rightarrow V \rightarrow \pi_{*}(\tilde{V}) \rightarrow \oplus_{i=1}^{g} \frac{\tilde{V}_{a_{i}} \oplus \tilde{V}_{b_{i}}}{F_{i}(\tilde{V})} \rightarrow 0
$$

is a torsion free sheaf on $X$ with $\operatorname{rk}(V)=\operatorname{rk}(\tilde{V})$. Conversely given any torsion-free sheaf on $X$, there is a GPB on $\tilde{X}$ which gives rise to it in this manner. The following theorem is from Bho92.

Theorem 2.12.2. Under the correspondence $\left(\tilde{V}, F_{i}(\tilde{V})\right) \mapsto V$, a generalized parabolic bundle of type $B$ gives rise to a vector bundle $V$ and conversely every vector bundle on $X$ arises from a GPB of type $B$ on $\tilde{X}$. This GPB of type $B$ is unique upto isomorphism.

\section{THETA DIVISORS FOR GENERALIZED PARABOLIC BUNDLES}

3.1. We extend the notion of theta divisors of vector bundles to theta divisors of generalized parabolic bundles.

3.2. We will say that a generalized parabolic bundle $\left(\tilde{V}, F_{i}(\tilde{V})\right)$ has a generalized parabolic theta divisor if there exists a GPB of type $B$ with $\operatorname{rk}(\tilde{L})=1$ such that

$$
\operatorname{Hom}\left(\left(\tilde{L}, F_{i}(\tilde{L})\right),\left(\tilde{V}, F_{i}(\tilde{V})\right)\right)=0 .
$$


3.3. If no such line bundle exists for a $\left(\tilde{V}, F_{i}(\tilde{V})\right)$, then every GPB of type $B$ and rank one admits a non-zero map to $\left(\tilde{V}, F_{i}(\tilde{V})\right)$. This is similar to the situation for vector bundles.

3.4. We now explain why the nomenclature "theta divisor" is appropriate. To do this we need to explicate the condition in the definition. We may do this in $\operatorname{Pic}(X)$, rather than in the scheme of GPB line bundles of type $B$ on $\tilde{X}$ (which is a $\mathbb{P}^{1}$-bundle on the compactification of Pic $(X))$.

Suppose $\left(\tilde{L}, F_{i}(\tilde{L})\right)$ is a GPB of type $B$ on $\tilde{X}$ with $\mathrm{rk}(\tilde{L})=1$ (so it gives rise to a line bundle $L$ on $X$. Moreover $F_{i}(\tilde{L}) \subset L_{a_{i}} \oplus L_{b_{i}}$ is the graph of some isomorphism $L_{a_{i}} \rightarrow L_{b_{i}}$. Such an isomorphism is given by a non-zero scalar, say $\lambda_{i}$.

Now suppose $\left(\tilde{V}, F_{i}(\tilde{V})\right)$ is a GPB of type $B$ and suppose that we have a morphism of GPB's $\left(\tilde{L}, F_{i}(\tilde{L})\right) \rightarrow\left(\tilde{V}, F_{i}(\tilde{V})\right)$. Then we have a commutative diagram

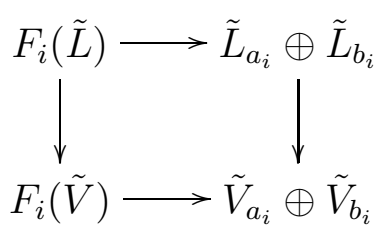

Let $F_{i}(\tilde{V})$ be the graph of an isomorphism $A_{i}: \tilde{V}_{a_{i}} \rightarrow \tilde{V}_{b_{i}}$, for $1 \leq i \leq g$. Then the commutativity of the diagram forces the condition

$$
\prod_{i=1}^{g} \operatorname{det}\left(A_{i}-\lambda_{i} I\right)=0
$$

This is a divisorial condition in the space of GPB bundles of type $B$ and rank one and the above equation is the equation of the theta divisor (when it is not all of $\operatorname{Pic}(X))$.

\section{A ZERO LEMMA}

4.1. In this section we prove a couple of preparatory lemmas for the next section.

Proposition 4.1.1. Let $\left(\tilde{V}, F_{i}(\tilde{V})\right)$ be a semistable $G P B$ of type $B$ on $\tilde{X}$, assume $\operatorname{deg}(\tilde{V})=0$. Suppose $\left(\tilde{L}, F_{i}(\tilde{L})\right)$ is a $G P B$ of type $B$ with $\operatorname{rk}(\tilde{L})=1$ and $\operatorname{deg}(\tilde{L})=1-g$. Suppose $\left(\tilde{L}, F_{i}(\tilde{L})\right) \rightarrow\left(\tilde{V}, F_{i}(\tilde{V})\right)$ is any non-zero map. Assume that the underlying morphism of $\mathcal{O}_{\tilde{X}}$-modules $\tilde{L} \rightarrow \tilde{V}$ vanishes at $d$ of the $2 g$ point $\left\{a_{i}, b_{i}\right\}_{1 \leq i \leq g}$. Then $d \leq 2 g-1$.

Proof. If the map $\tilde{L} \rightarrow \tilde{V}$ vanishes at $d$ points, then this map factors as $\tilde{L} \rightarrow \tilde{M}=$ $\mathcal{O}_{\mathbb{P}^{1}}(1-g+d) \rightarrow \tilde{V}$. Now give $\tilde{M}=\mathcal{O}_{\mathbb{P}^{1}}(1-g+d)$ the induced GPB structure (see 2.6 $F_{i}^{\text {ind }}(\tilde{M}) \subset \tilde{M}_{a_{i}} \oplus \tilde{M}_{b_{i}}$. Now we have

$$
\mu\left(\left(\tilde{M}, F_{i}(\tilde{M})\right)\right)=\operatorname{deg}(\tilde{M})+\sum_{i} \operatorname{dim} F_{i}(\tilde{M}) .
$$

So in any case $\mu\left(\left(\tilde{M}, F_{i}(\tilde{M})\right)\right) \geq 1-g+d$. On the other hand $\left(\tilde{V}, F_{i}(\tilde{V})\right)$ is a semistable GPB of type $B$. So its generalized parabolic slope is

$$
\mu\left(\left(\tilde{V}, F_{i}(\tilde{V})\right)\right)=\operatorname{deg}(\tilde{V})+g=g .
$$

Thus by semi-stability we see that

$$
1-g+d \leq \mu\left(\left(\tilde{M}, F_{i}(\tilde{M})\right)\right) \leq \mu\left(\left(\tilde{V}, F_{i}(\tilde{V})\right)\right)=g,
$$


that is, $1-g+d \leq g$. Hence $d \leq 2 g-1$. This proves the proposition.

\section{Theta Divisors for GPB Bundles of TyPE $B$}

5.1. Now we are ready to prove Theorem 1.3.1, Let us recall the statement.

Theorem 5.1.1. Let $\left(\tilde{V}, F_{i}(\tilde{V})\right)$ be a semistable GPB of type $B$ on $\tilde{X}$. Suppose that $\operatorname{deg}(\tilde{V})=0$ and $g \geq 2$. Then there exists a $G P B\left(\tilde{L}, F_{i}(\tilde{L})\right)$, with $\operatorname{deg}(\tilde{L})=1-g$ and $\operatorname{rk}(\tilde{L})=1$ such that

$$
\operatorname{Hom}\left(\left(\tilde{L}, F_{i}(\tilde{L})\right),\left(\tilde{V}, F_{i}(\tilde{V})\right)\right)=0 .
$$

In other words $\left(\tilde{V}, F_{i}(\tilde{V})\right)$ has a theta divisor.

Proof of Theorem 5.1.1. We will construct such a $\left(\tilde{L}, F_{i}(\tilde{L})\right)$ on $\tilde{X}$ with the asserted property. Since $\operatorname{deg}(\tilde{L})=1-g$, we take $\tilde{L}=\mathcal{O}_{\mathbb{P}^{1}}(1-g)$. So we need to construct a GPB structure on $\tilde{L}$ such that there are no maps $\left(\tilde{L}, F_{i}(\tilde{L})\right) \rightarrow\left(\tilde{V}, F_{i}(\tilde{V})\right)$. Observe that by the Riemann-Roch theorem,

$$
\chi(\tilde{V}(g-1))=\operatorname{rk}(\tilde{V})(g-1)+\operatorname{rk}(\tilde{V})(1-0) \neq 0,
$$

so that $\operatorname{Hom}(\tilde{L}, \tilde{V}) \neq 0$.

Let $A_{i}: \tilde{V}_{a_{i}} \rightarrow \tilde{V}_{b_{i}}$ be the isomorphism whose graph is $F_{i}(\tilde{V}) \subset \tilde{V}_{a_{i}} \oplus \tilde{V}_{b_{i}}$. Choose, for $1 \leq i \leq g$, non-zero scalars $\lambda_{i} \in k^{*}$ such that $\lambda_{i}$ is not an eigenvalue of $A_{i}$. Let $F_{i}(\tilde{L})$ be the graph of the isomorphism $\lambda_{i}: \tilde{L}_{a_{i}} \rightarrow \tilde{L}_{b_{i}}$. Then we claim that

$$
\operatorname{Hom}\left(\left(\tilde{L}, F_{i}(\tilde{L})\right),\left(\tilde{V}, F_{i}(\tilde{V})\right)\right)=0 .
$$

Suppose this is not the case. Then there is a non-zero map

$$
f:\left(\tilde{L}, F_{i}(\tilde{L})\right) \rightarrow\left(\tilde{V}, F_{i}(\tilde{V})\right) .
$$

Consider the underlying map of $\mathcal{O}_{\tilde{X}}$-modules $f: \tilde{L} \rightarrow \tilde{V}$. By Proposition 4.1.1 we know that the map $f$ can vanishes at no more than $2 g-1$ of the $2 g$ points $a_{i}, b_{i}$ for $1 \leq i \leq g$. Assume if possible that the map $f$ vanishes at all except one points, say, $a_{1}$, and by assumption we see that the map $f$ must be vanishing at $b_{1}$.

As $\left(\tilde{L}, F_{i}(\tilde{L})\right) \rightarrow\left(\tilde{V}, F_{i}(\tilde{V})\right)$ is a morphism of GPB structures, the diagram

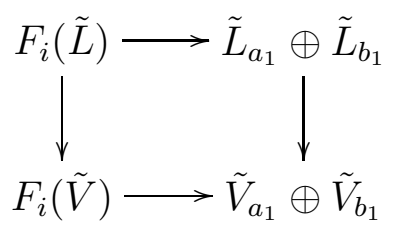

commutes. On the other hand, the horizontal arrows are inclusions and the component $\tilde{L}_{a_{1}} \rightarrow \tilde{V}_{a_{1}}$ is non-zero. So the first vertical arrow must also be non-zero. Now the composite map $F_{1}(\tilde{L}) \rightarrow \tilde{V}_{a_{1}} \oplus \tilde{V}_{b_{1}}$ is also non-zero. This is because $F_{1}(\tilde{L})$ is the graph of the isomorphism $\lambda_{1}: \tilde{L}_{a_{1}} \rightarrow \tilde{L}_{b_{1}}$ and so the image of $F_{1}(\tilde{L})$ in the direct sum is not in the kernel, $L_{b_{1}}$, of $\tilde{L}_{a_{1}} \oplus \tilde{L}_{b_{1}} \rightarrow \tilde{V}_{a_{1}} \oplus \tilde{V}_{b_{1}}$. So we deduce, by commutativity of the diagram, that $F_{1}(\tilde{L}) \rightarrow F_{1}(\tilde{V})$ is also non-zero.

Let $\tilde{\ell}$ be a basis of $F_{1}(\tilde{L})$ (note that this is a one dimensional space). As the diagram commutes, the image of $\tilde{\ell}$ is, on one hand, equal to $\tilde{\ell} \mapsto\left(\tilde{\ell}, \lambda_{1} \tilde{\ell}\right) \mapsto(\tilde{v}, 0)$. While on the other hand, it is given by $\tilde{\ell} \mapsto \tilde{w} \mapsto\left(\tilde{u}, A_{1} \tilde{u}\right)$ where $F_{1}(\tilde{V})$ is the graph of $A_{1}: \tilde{V}_{a_{1}} \rightarrow \tilde{V}_{b_{1}}$. So we must have $(\tilde{v}, 0)=\left(\tilde{u}, A_{1} \tilde{u}\right)$. Hence we must have $\tilde{v}=\tilde{u}$ and $A_{1} \tilde{u}=0$. But $A_{1}: \tilde{V}_{a_{1}} \rightarrow \tilde{V}_{b_{1}}$ is an isomorphism. So $A_{1} \tilde{u}=0$ gives $\tilde{u}=0$. 
Hence the image of $\tilde{\ell}$ under the composite $F_{1}(\tilde{L}) \rightarrow F_{1}(\tilde{V}) \rightarrow \tilde{V}_{a_{1}} \oplus \tilde{V}_{b_{1}}$ is zero. But this is a contradiction. Hence our assumption that $f: \tilde{L} \rightarrow \tilde{V}$ vanishes at exactly one point must be wrong.

So we see that $f$ must be non-vanishing at two or more points and further, the same argument as above shows that the set of points at which it is non-vanishing must contain a pair of the form $a_{i}, b_{i}$ for some $i$.

Thus we can assume that $\tilde{L} \rightarrow \tilde{V}$ induces a non-zero map at the stalks at a pair of points, say $a_{1}, b_{1}$ (after a renumbering these points if required), with a torsion-free cokernel. Thus $\tilde{L} \rightarrow \tilde{V}$ is a bundle map at $a_{1}, b_{1}$. So we have an inclusion of fibres $\tilde{L}_{a_{1}} \subset \tilde{V}_{a_{1}}$ and similarly at the point $b_{1}$. So we can identify the fibres of $\tilde{L}$ at these points as subspaces of the fibres of $\tilde{V}$ at the corresponding points. Now the fact that we have a map of generalized parabolic bundles means that we have a commutative diagram

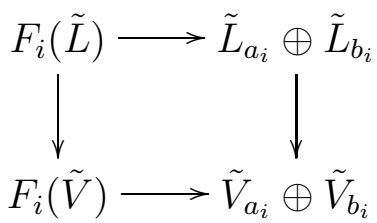

commutes and $\tilde{L}_{a_{i}} \subset \tilde{V}_{a_{i}}$ etc. But this is not possible: as $F_{i}(\tilde{L})$ is the graph of $\lambda_{i}$ and $F_{i}(\tilde{V})$ is the graph of $A_{i}$, so the commutativity forces $\lambda_{i}$ to be an eigenvalue of $A_{i}$ and as $\lambda_{i}$ is not an eigenvalue of $A_{i}$, by choice, so we have a contradiction. This proves the assertion.

Corollary 5.1.3. Let $V$ be a semistable bundle of degree zero on the Castelnuovo curve $X$ of genus $g \geq 2$. Then $V$ has a theta divisor, that is there exists a line bundle on $X$, of degree $1-g$, such that $\operatorname{Hom}(L, V)=0$.

Proof. Let $\left(\tilde{V}, F_{i}(\tilde{V})\right)$ be the GPB bundle of type $B$ on $\tilde{X}$ associated to $V$ by 2.12 , Then by the theorem, there exists $\left(\tilde{L}, F_{i}(\tilde{L})\right)$ such that

$$
\operatorname{Hom}\left(\left(\tilde{L}, F_{i}(\tilde{L})\right),\left(\tilde{V}, F_{i}(\tilde{V})\right)\right)=0 .
$$

. We claim that there is a natural map

$$
\operatorname{Hom}\left(\left(\tilde{L}, F_{i}(\tilde{L})\right),\left(\tilde{V}, F_{i}(\tilde{V})\right)\right) \rightarrow \operatorname{Hom}(L, V) .
$$

To prove this we observe that we have a commutative diagram with exact rows

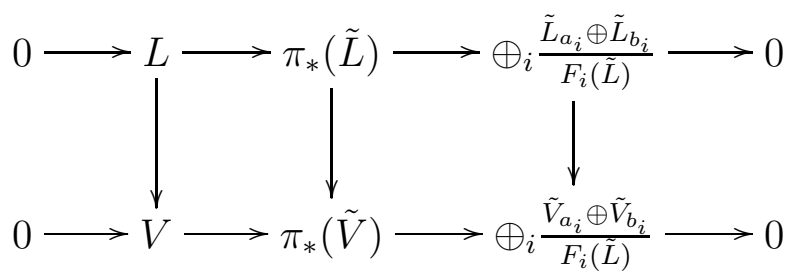

In the diagram, the right most vertical arrow is the direct sum of the arrows, one for each $i$, for $1 \leq i \leq g$. The arrow for each $i$ is given using the fact that the map $F_{i}(\tilde{L}) \rightarrow F_{i}(\tilde{V})$. The middle arrow is the push-forward of the arrow $\tilde{L} \rightarrow \tilde{V}$ underlying the map $\left(\tilde{L}, F_{i}(\tilde{L})\right) \rightarrow\left(\tilde{V}, F_{i}(\tilde{V})\right)$. Thus we have constructed the arrow $L \rightarrow V$ given a map of $\left(\tilde{L}, F_{i}(\tilde{L})\right) \rightarrow\left(\tilde{V}, F_{i}(\tilde{V})\right)$.

We claim that $\operatorname{Hom}\left(\left(\tilde{L}, F_{i}(\tilde{L})\right),\left(\tilde{V}, F_{i}(\tilde{V})\right)\right) \rightarrow \operatorname{Hom}(L, V)$ is onto. So suppose that we have a map $f: L \rightarrow V$ we have to construct a corresponding map of 
$\left(\tilde{L}, F_{i}(\tilde{L})\right) \rightarrow\left(\tilde{V}, F_{i}(\tilde{V})\right)$ which gives rise to it in the above fashion. Pulling back the map $f$ by $\pi$, we get a $\pi^{*}(f): \pi^{*}(L) \rightarrow \pi^{*}(V)$ and noting that $\pi^{*}(L)=\tilde{L}$ and $\pi^{*}(V)=\tilde{V}$, we deduce that we have a map

$$
\tilde{f}: \tilde{L} \rightarrow \tilde{V}
$$

Now we have to verify that we also have a map of parabolic data at the points $a_{i}, b_{i}$ for $1 \leq i \leq g$. But as the map $\tilde{f}$ which we just constructed is pulled back from $X$, it comes equipped with gluing data at the $a_{i}, b_{i}$ for $1 \leq i \leq g$, which gives maps of the parabolic data at the points over the nodes of $X$. Further as $L, V$, are locally free sheaves on $X$, by [Bho92], we know that $L, V$ arise from unique generalized parabolic bundles on $\tilde{X}$ of type $B$. Thus, we see that we have constructed a non-zero map of generalized parabolic bundles $\left(\tilde{L}, F_{i}(\tilde{L})\right) \rightarrow\left(\tilde{V}, F_{i}(\tilde{V})\right)$, and this is a contradiction.

Thus we have completed the proof of the corollary.

5.2. Now we are ready to complete the proof of Theorem 1.1.1. We recall what we have to prove:

Theorem 5.2.1. Suppose $\mathfrak{X} \rightarrow \operatorname{Spec}(k[[t]])$ is flat, proper family of curves with smooth generic fibre $\mathfrak{X}_{\eta}$ and the special fibre $\mathfrak{X}_{0}=X$ is a Castelnuovo curve of arithmetic genus $g \geq 2$. Suppose $V$ is a semistable vector bundle on $X_{\eta}$ of degree zero which extends as a semistable vector bundle on $\mathfrak{X}_{0}$. Then $V$ has a theta divisor.

Proof of Theorem 5.2.1. Now we are ready to prove Theorem 5.2.1. Suppose $\mathfrak{X} \rightarrow$ $\operatorname{Spec}(k[[t]])$ is a flat family of curves with smooth, projective generic fibre of genus $g$, denoted $\mathfrak{X}_{\eta}$ and the special fibre $\mathfrak{X}_{0}=X$ is a Castelnuovo curve of genus $g$. Suppose $V_{\eta}$ is a vector bundle of degree zero on $\mathfrak{X}_{\eta}$ which extends to a vector bundle $V$ on $X$. By Theorem 1.2.1, there exists a line bundle $L$ on $X$, of degree $1-g$ on $X$ such that $\operatorname{Hom}(L, V)=0$. As $\operatorname{Ext}^{2}\left(\mathcal{O}_{X}, \mathcal{O}_{X}\right)=0, L$ extends to a line bundle $L_{\eta}$ on $\mathfrak{X}_{\eta}$ and by the semi-continuity theorem we see that $\operatorname{Hom}\left(L_{\eta}, V_{\eta}\right)=0$ as $\operatorname{Hom}(L, V)=0$. This proves the theorem.

5.3. Now Corollary 1.1.2 can also be established.

Corollary 5.3.1. Let $X / k$ be a general smooth, projective curve of genus $g \geq 2$. Let $V$ be a semistable vector bundle on $X$ which extends to a semistable vector bundle on some Castelnuovo degeneration of $X$. Then $V$ admits a theta divisor.

Proof of Corollary [5.3.1. By [DM69] we know that a general curve of genus $g \geq 2$ admits a Castelnuovo degeneration and indeed many such degenerations. Thus if $V$ is a vector bundle which extends to some Castelnuovo degeneration, then Theorem 5.2 .1 applies and we are done.

\section{REFERENCES}

[Bho92] Usha Bhosle, Generalised parabolic bundles and applications to torsionfree sheaves on nodal curves, Ark. Mat. 30 (1992), no. 2, 187-215.

[Bho96] Usha N. Bhosle, Generalized parabolic bundles and applications. II, Proc. Indian Acad. Sci. Math. Sci. 106 (1996), no. 4, 403-420.

[DM69] P. Deligne and D. Mumford, The irreducibility of the space of curves of given genus, Inst. Hautes Études Sci. Publ. Math. (1969), no. 36, 75-109.

[JM] Kirti Joshi and V. B. Mehta, Vectors bundles with theta divisors II: Vector bundles on nodal curves, Under preparation. 
[Ray82] Michel Raynaud, Sections des fibrés vectoriels sur une courbe, Bull. Soc. Math. France 110 (1982), no. 1, 103-125.

[Ses82] C. S. Seshadri, Fibrés vectoriels sur les courbes algébriques, Astérisque, vol. 96, Société Mathématique de France, Paris, 1982, Notes written by J.-M. Drezet from a course at the École Normale Supérieure, June 1980.

Math. Department, University of Arizona, 617 N Santa Rita, Tucson 85721-0089, USA.

E-mail address: kirti@math.arizona.edu

School of Mathematics, Tata Institute of Fundamental Research, Mumbai, India. E-mail address: vikram@math.tifr.res.in 